\title{
Development of an Analytical Method for the Determination of Arsenic in Urine by Gas Chromatography-mass Spectrometry for Biological Monitoring of Exposure to Inorganic Arsenic
}

\author{
Akito TAKeuchi ${ }^{1}$, Akira NAmera ${ }^{2}$, Yaeko Kawasumi ${ }^{3}$, Tsutoshi Imanaka ${ }^{4}$, \\ Norihiro SAKUI ${ }^{5}$, Hirokazu OTA ${ }^{1}$, Yoko ENDO ${ }^{6}$, Kimiaki Sumino ${ }^{1}$ and Ginji ENDO ${ }^{7}$
}

${ }^{1}$ Osaka Occupational Health Service Center, Japan Industrial Safety and Health Association, Japan, ${ }^{2}$ Department of Forensic Medicine, Institute of Biomedical and Health Sciences, Hiroshima University, Japan, ${ }^{3}$ Occupational Health Research and Development Center, Japan Industrial Safety and Health Association, Japan, ${ }^{4}$ Fukushima Factory, GL Sciences Inc., Japan, ${ }^{5}$ Agilent Technologies, Japan, ${ }^{6}$ Research Center for Occupational Poisoning, Kansai Rosai Hospital, Japan Labour Health and Welfare Organization, Japan and ${ }^{7}$ Department of Preventive Medicine and Environmental Health, Graduate School of Medicine, Osaka City University, Japan

\begin{abstract}
Development of an Analytical Method for the Determination of Arsenic in Urine by Gas Chromatography-mass Spectrometry for Biological Monitoring of Exposure to Inorganic Arsenic: Akito TAKEUCH, et al. Osaka Occupational Health Service Center, Japan Industrial Safety and Health Association-Objectives: The purpose of this study was to develop an analytical method for the simultaneous determination of inorganic arsenic [As(III) and $\mathrm{As}(\mathrm{V})]$ and monomethylarsonic acid (MMA) in urine by gas chromatography-mass spectrometry (GC-MS) for the biological monitoring of exposure to inorganic arsenic. Methods: Arsenic compounds (after reduction of arsenic to the trivalent state) were derivatized with 2,3dimercapto-1-propanol and then analyzed using a GC-MS. The proposed method was validated according to the US Food and Drug Administration guidelines. The accuracy of the proposed method was confirmed by analyzing Standard Reference Material (SRM) 2669 (National Institute of Standards and Technology). Results: Calibration curves showed linearity in the range 1-100 $\mu \mathrm{g} / /$ for each of the arsenic species, with correlation coefficients of $>0.999$. For each of the arsenic species, the limits of detection and quantification were $0.2 \mu \mathrm{g} / /$ and $1 \mu \mathrm{g} / /$, respectively. The recoveries were $96-100 \%, 99-102 \%$ and $99-112 \%$ for $\mathrm{As}(\mathrm{III})$, $\mathrm{As}(\mathrm{V})$ and MMA, respectively. Intraday accuracy and precision were $82.7-99.8 \%$ and $0.9-7.4 \%$, respectively.
\end{abstract}

Received May 10, 2012; Accepted Aug 28, 2012

Published online in J-STAGE Oct 4, 2012

Correspondence to: A. Takeuchi, Osaka Occupational Health Service Center, Japan Industrial Safety and Health Association, 2-3-8 Tosabori, Nishi-ku, Osaka 550-0001, Japan

(e-mail: a-takeuchi@jisha.or.jp)
Interday accuracy and precision were $81.3-100.0 \%$ and $0.8-9.9 \%$, respectively. The analytical values of SRM 2669 obtained by the proposed method were sufficiently accurate. Conclusions: The proposed method overcame the disadvantages of high-performance liquid chromatography with inductively coupled plasma mass spectrometry. It was a robust, selective and cost-effective method suitable for routine analyses and could be useful for the biological monitoring of occupational exposure to inorganic arsenic.

(J Occup Health 2012; 54: 434-440)

Key words: 2,3-Dimercapto-1-propanol, Arsenate, Arsenite, Gas chromatography-mass spectrometry, Monomethylarsonic acid, Urine

Arsenic is widely distributed in the environment. Pollution due to arsenic worldwide has become a serious problem and has caused various adverse effects on human health ${ }^{1)}$. Significant occupational exposure to arsenic exists in several industries, such as non-ferrous smelting, electronics and wood preservation ${ }^{1)}$. Such exposure is mainly in the form of inorganic arsenic (iAs), including arsenite [As(III)] and arsenate $[\mathrm{As}(\mathrm{V})]^{2,3)}$. Arsenic and inorganic arsenic compounds have been classified as Group 1 (carcinogenic to humans) compounds by the International Agency for Research on Cancer (IARC) because they cause cancers of the skin, bladder and lungs, and there is limited evidence that they may also cause cancers of the kidney, liver and prostate ${ }^{4}$.

iAs is methylated to monomethylarsonic acid (MMA) and dimethylarsinic acid (DMA) in the human body ${ }^{1-4)}$. The American Conference of Governmental 
and Industrial Hygienists (ACGIH) $)^{2)}$ and the Deutsche Forschungsgemeinschaft $(\mathrm{DFG})^{3)}$ recommend the sum of iAs, MMA and DMA concentrations as the biological exposure value for iAs exposure. However, large amounts of DMA and arsenobetaine (AsBe) and small amounts of MMA and iAs have been observed in the urine of Japanese individuals who habitually ingest seafood without occupational exposure to iAs because seafood (including seaweeds) contains high levels of organoarsenic compounds, such as DMA, $\mathrm{AsBe}$, arsenosugars and arsenocholine ${ }^{5)}$. Recently, Hata et al. reported that the sum of iAs and MMA concentrations in urine was more suitable than that of iAs, MMA and DMA concentrations in urine for the biological monitoring of occupational iAs exposure of workers who habitually consume seafood ${ }^{5}$. The Ministry of Health, Labour and Welfare of Japan also defined the sum of iAs and MMA concentrations in urine as the iAs exposure indicator ${ }^{6}$. Therefore, a rapid and simple analysis for only iAs and MMA is more effective than speciation analysis for the biological monitoring of occupational exposure to iAs.

Currently, high-performance liquid chromatography with inductively coupled plasma mass spectrometry (HPLC-ICP-MS) is the mainstay of speciation analyses of arsenic in biological samples because of its sensitivity and effectiveness ${ }^{5,7)}$. However, it is a remarkably expensive and complicated system, and hence it is not commonly used in many laboratories. Moreover, its operation requires skilled techniques and high running costs.

The aim of the present study was to develop and validate a gas chromatography-mass spectrometry (GC-MS) method for the simultaneous determination of iAs and MMA in urine. To obtain the sensitivity required for biological monitoring, we modified the previous GC-flame photometric detection method using 2,3-dimercapto-1-propanol (also known as British Anti-Lewisite: BAL) as the derivatizing reagent ${ }^{8}$. We used GC-MS equipped with a capillary column, because it has a high capability of separation, high sensitivity and high efficiency for confirmatory identification of compounds. Another required modification was optimization of the procedure for sample preparation, including optimization of reaction conditions, scale-down of the sample volume and change of extraction solvent. The proposed method overcame the disadvantages of HPLC-ICP-MS. It was a robust, selective and cost-effective method suitable for routine analyses.

\section{Materials and Methods}

Materials

Sodium metaarsenite was purchased from SigmaAldrich Co. (St Louis, MO, USA). Disodium hydro- genarsenate heptahydrate and BAL were obtained from Wako Pure Chemical Industries, Ltd. (Osaka, Japan). Methylarsonic acid/water solution $(1,050 \mu \mathrm{g} / \mathrm{m} l)$ was procured from Tri Chemical Laboratories Inc. (Yamanashi, Japan). Hydrochloric acid ( $\mathrm{HCl})$, tin (II) chloride dihydrate $\left(\mathrm{SnCl}_{2} \cdot 2 \mathrm{H}_{2} \mathrm{O}\right)$, potassium iodide (KI), dichloromethane (DCM) and hexadecane (C16) were of analytical grade or better (for atomic adsorption spectrometry or for analysis of poisonous metal). Water was purified with an Elix 5 system (Millipore, Bedford, MA, USA). Standard Reference Material (SRM) 2669 (arsenic species in frozen human urine) was purchased from the National Institute of Standards and Technology (NIST, Gaithersburg, MD, USA).

$\mathrm{SnCl}_{2}$ solution was prepared by dissolving $\mathrm{SnCl}_{2} .2 \mathrm{H}_{2} \mathrm{O}$ in $\mathrm{HCl}(50 \mathrm{mg} / \mathrm{ml})$. KI solution [20\% $(\mathrm{w} / \mathrm{w})]$ and BAL solution $[0.2 \%(\mathrm{v} / \mathrm{v})]$ were prepared by dissolving $\mathrm{KI}$ and BAL, respectively, in water. The extraction solution was DCM containing C16 $(10 \mu \mathrm{g} / \mathrm{ml})$ as an internal standard (IS). Standard stock solutions of As(III), As(V) and MMA were prepared in water $(100 \mathrm{mg} / \mathrm{l}$ as As) and stored in PFA bottles at $4^{\circ} \mathrm{C}$.

Urine samples were collected from healthy adult volunteers. These volunteers were not occupationally exposed to arsenic and were under dietary restriction of seafood. Informed consent had been obtained from the volunteers before collection of urine. This study was approved by the Ethics Committees of the Graduate School of Medicine, Osaka City University (approval number 2367).

\section{Instruments}

The GC-MS system used was a 7890A gas chromatograph equipped with a 5975C inert XL mass spectrometer (Agilent Technologies, Palo Alto, CA, USA). The column was a $30 \mathrm{~m} \times 0.25 \mathrm{~mm}$ ID InertCap 5MS/NP capillary column with a $0.25-\mu \mathrm{m}$ film thickness (GL Sciences Inc., Tokyo, Japan). Helium was used as the carrier gas at a flow rate of $1.0 \mathrm{~m} / / \mathrm{min}$. The temperatures of the injection port and the transfer line were set at 250 and $280^{\circ} \mathrm{C}$, respectively. The oven temperature was set at $70^{\circ} \mathrm{C}$ for 1 minute and then increased to $280^{\circ} \mathrm{C}$ at a rate of $10^{\circ} \mathrm{C} / \mathrm{min}$. Samples $(2 \mu l)$ were injected in the pulsed splitless mode (pulse pressure, $25 \mathrm{psi}$; pulse time, 1 minute; purge activation time, 0.9 minutes). The mass spectrometer was operated in the electron impact (EI) mode at an electron energy of $70 \mathrm{eV}$. The ion source and quadrupole analyzer were maintained at 230 and $150^{\circ} \mathrm{C}$, respectively. Data were obtained in the selected ion monitoring (SIM)/scan mode. The ions selected for SIM were m/z 197 and 212 for BAL-MMA, $\mathrm{m} / \mathrm{z} 226$ and 183 for $\mathrm{C} 16$ and m/z 212 and 165 for 

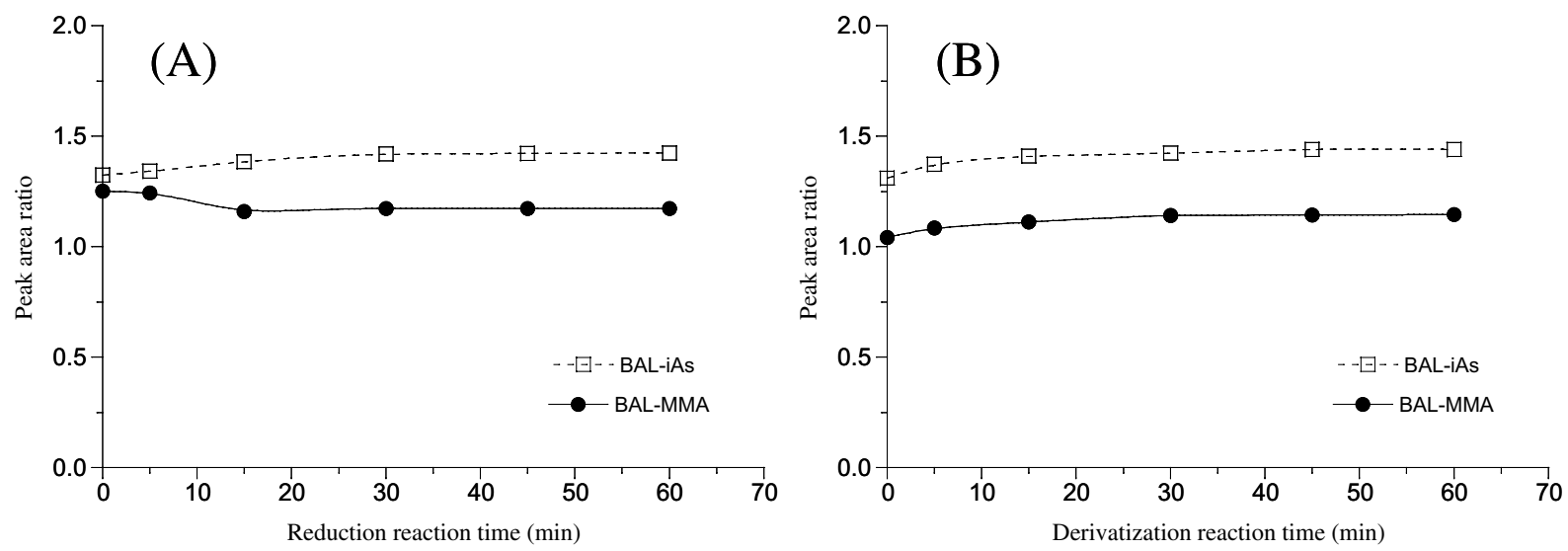

Fig. 1. Effects of the (A) reduction reaction time and (B) derivatization reaction time on the production of the derivatives of inorganic arsenic (BAL-iAs) and monomethylarsonic acid (BAL-MMA).

BAL-iAs, in which the former was selected as the quantifier ion and the latter as the qualifier ion. To confirm the mass fragmentation of the derivatives, data were obtained in scan mode with a scan range from $\mathrm{m} / \mathrm{z} 40$ to 250 .

\section{Sample preparation}

Urine $(2 \mathrm{ml})$ was placed in a glass test tube. $\mathrm{SnCl}_{2}$ $(0.4 \mathrm{ml})$ and $\mathrm{KI}(0.2 \mathrm{ml})$ solutions were added to the tube, which was vortex-mixed (10 seconds) and then allowed to stand for 30 minutes at room temperature. BAL solution $(0.2 \mathrm{ml})$ was added to the tube, which was vortex-mixed (10 seconds) and then allowed to stand for 30 minutes at room temperature. Extraction solution $(0.5 \mathrm{ml})$ was added to the tube and shaken gently for 1 minute to avoid emulsion formation. The mixture was centrifuged at 3,000 rpm for 10 minutes, and a $2-\mu l$ aliquot of the extraction solution layer was injected into the GC-MS system.

\section{Method validation}

Method validation was conducted according to the US Food and Drug Administration (FDA) guidelines ${ }^{9)}$. To plot calibration curves, urine samples spiked with As(III) or As(V) and MMA at six concentrations (matrix-matched standard) ranging from 1 to $100 \mu \mathrm{g} / l$ were prepared in triplicate, and the prepared samples were analyzed using the procedure for sample preparation described above. Calibration curves were obtained by plotting the peak area ratio of BAL derivatives of $\mathrm{As}(\mathrm{III}), \mathrm{As}(\mathrm{V})$ or MMA to IS against their respective concentrations. The reproducibility, which was defined as precision, of the developed method was evaluated by analyzing urine samples containing three concentrations (1, 25 and $100 \mu \mathrm{g} / l)$ of As(III) or As(V) and MMA on the same day (five replicates; intraday reproducibility) and over three consecutive days (five replicates; interday reproducibility). Recovery was determined by comparing the responses of the BAL derivative of $\mathrm{As}(\mathrm{III}), \mathrm{As}(\mathrm{V})$ or MMA in spiked urine samples with those of water standards subjected to the same procedure. The limit of detection (LOD) and limit of quantification (LOQ) were defined as the amounts of $\mathrm{As}(\mathrm{III}), \mathrm{As}(\mathrm{V})$ or MMA in urine that corresponded to 3 and 10 times the baseline noise, respectively. Finally, the accuracy of the proposed method was confirmed using SRM 2669. The obtained results and certified values were compared according to application note 1 of the Institute for Reference Materials and Measurements $(\text { IRMM })^{10)}$.

\section{Results}

Optimization of reaction conditions

To determine the optimal reaction conditions, we examined the influences of the reduction reaction time and the derivatization reaction time using urine samples spiked with $100 \mu \mathrm{g} / l$ each of $\mathrm{As}(\mathrm{V})$ and MMA. When the reduction reaction time ranged from 0 to 60 minutes with a derivatization reaction time of 30 minutes, the reduction reaction was complete within a short time, and the BAL-iAs/IS and BAL-MMA/IS peak area ratios remained at a plateau until 60 minutes [Fig. 1(A)]. When the derivatization reaction time ranged from 0 to 60 minutes with a reduction reaction time of 30 minutes, the BAL-iAs/IS and BAL-MMA/IS peak area ratios attained maximum area ratios after 30 minutes and remained constant for 60 minutes [Fig. 1(B)].

\section{Validation}

Calibration curves showed linearity in the range $1-100 \mu \mathrm{g} / l$ for each of the arsenic species, with correlation coefficients of $>0.999$. For each of the arsenic 
Table 1. Range of linearity and correlation coefficient of the proposed method

\begin{tabular}{lccccc}
\hline & \multirow{2}{*}{$\begin{array}{c}\text { Detection limit } \\
(\mu \mathrm{g} / l)\end{array}$} & $\begin{array}{c}\text { Range of linearity } \\
(\mu \mathrm{g} / l)\end{array}$ & \multicolumn{2}{c}{ Linearity } & Correlation coefficient \\
\cline { 4 - 5 } & 0.2 & $1-100$ & 0.0149 & 0.003 & 1.000 \\
$\mathrm{As}(\mathrm{III})$ & 0.2 & $1-100$ & 0.0146 & 0.004 & 1.000 \\
$\mathrm{As}(\mathrm{V})$ & 0.2 & $1-100$ & 0.0122 & -0.005 & 0.999 \\
$\mathrm{MMA}$ & & 100 & Intercept & \\
\hline
\end{tabular}

As(III): arsenite. As(V): arsenate. MMA: monomethylarsonic acid.

Table 2. Intra- and interday coefficients of variation of the proposed method

\begin{tabular}{|c|c|c|c|c|c|c|c|c|}
\hline \multirow{2}{*}{$\begin{array}{l}\text { Spiked urine } \\
\text { concentration } \\
(\mu \mathrm{g} / l)\end{array}$} & \multicolumn{2}{|c|}{ Recovery $(\mathrm{n}=5)$} & \multicolumn{3}{|c|}{ Intraday $(\mathrm{n}=5)$} & \multicolumn{3}{|c|}{ Interday $(\mathrm{n}=15)$} \\
\hline & $\begin{array}{c}\text { Mean } \pm \text { SD } \\
(\%)\end{array}$ & $\operatorname{RSD}(\%)$ & $\begin{array}{c}\text { Mean } \pm \mathrm{SD} \\
(\mu \mathrm{g} / l)\end{array}$ & RSD (\%) & $\begin{array}{c}\text { Accuracy } \\
(\%)\end{array}$ & $\begin{array}{c}\text { Mean } \pm \mathrm{SD} \\
(\mu \mathrm{g} / l)\end{array}$ & $\operatorname{RSD}(\%)$ & $\begin{array}{c}\text { Accuracy } \\
(\%)\end{array}$ \\
\hline \multicolumn{9}{|l|}{ As(III) } \\
\hline 1 & $100 \pm 7.4$ & 7.4 & $0.90 \pm 0.07$ & 7.4 & 90.3 & $0.90 \pm 0.04$ & 4.8 & 89.5 \\
\hline 25 & $97 \pm 0.9$ & 0.9 & $24.14 \pm 0.23$ & 0.9 & 96.5 & $24.28 \pm 0.20$ & 0.8 & 97.1 \\
\hline 100 & $96 \pm 1.9$ & 2.0 & $99.79 \pm 2.03$ & 2.0 & 99.8 & $99.95 \pm 1.53$ & 1.5 & 100.0 \\
\hline \multicolumn{9}{|l|}{$\mathrm{As}(\mathrm{V})$} \\
\hline 1 & $102 \pm 5.7$ & 5.6 & $0.91 \pm 0.05$ & 5.6 & 91.1 & $0.91 \pm 0.09$ & 9.9 & 90.9 \\
\hline 25 & $99 \pm 1.2$ & 1.2 & $24.21 \pm 0.30$ & 1.2 & 96.8 & $24.33 \pm 0.34$ & 1.4 & 97.3 \\
\hline 100 & $99 \pm 3.1$ & 3.1 & $99.66 \pm 3.09$ & 3.1 & 99.7 & $99.97 \pm 2.18$ & 2.2 & 100.0 \\
\hline \multicolumn{9}{|l|}{ MMA } \\
\hline 1 & $107 \pm 6.9$ & 6.4 & $0.83 \pm 0.05$ & 6.4 & 82.7 & $0.81 \pm 0.05$ & 6.2 & 81.3 \\
\hline 25 & $99 \pm 1.1$ & 1.1 & $23.17 \pm 0.25$ & 1.1 & 92.7 & $23.47 \pm 0.56$ & 2.4 & 93.9 \\
\hline 100 & $112 \pm 3.0$ & 2.7 & $99.36 \pm 2.71$ & 2.7 & 99.4 & $99.78 \pm 2.80$ & 2.8 & 99.8 \\
\hline
\end{tabular}

$\mathrm{As}(\mathrm{III})$ : arsenite. $\mathrm{As}(\mathrm{V})$ : arsenate. MMA: monomethylarsonic acid. RSD: relative standard deviation.

Table 3. Results for arsenic species in NIST SRM 2669 (Levels I and II) ${ }^{\mathrm{a}}$

\begin{tabular}{lcclcc}
\hline \multirow{2}{*}{ Species } & \multicolumn{2}{c}{ Level I } & & \multicolumn{2}{c}{ Level II } \\
\cline { 2 - 3 } \cline { 5 - 6 } & $\begin{array}{c}\text { This } \\
\text { method }^{\mathrm{b}}\end{array}$ & Certified $^{\mathrm{c}}$ & & $\begin{array}{c}\text { This } \\
\text { method }^{\mathrm{b}}\end{array}$ & Certified $^{\mathrm{c}}$ \\
\hline As(III) & - & $1.47 \pm 0.10$ & - & $5.03 \pm 0.31$ \\
As(V) & - & $2.41 \pm 0.30$ & - & $6.16 \pm 0.95$ \\
iAs & $3.9 \pm 0.0$ & - & & $11.4 \pm 0.1$ & - \\
MMA & $2.4 \pm 0.4$ & $1.87 \pm 0.39$ & & $6.9 \pm 0.4$ & $7.18 \pm 0.56$ \\
\hline
\end{tabular}

${ }^{\mathrm{a}}$ Concentrations in $\mu \mathrm{g} / \mathrm{l}$ as As. ${ }^{\mathrm{b}}$ Values are expressed as the mean $\pm \mathrm{SD}(\mathrm{n}=3) .{ }^{\mathrm{c}}$ Values are expressed as the certified value \pm the expanded uncertainty (95\% confidence interval). As(III): arsenite. As(V): arsenate. MMA: monomethylarsonic acid. iAs: inorganic arsenic. NIST: National Institute of Standards and Technology. SRM: Standard Reference Material. species, LOD and LOQ were $0.2 \mu \mathrm{g} / l$ and $1 \mu \mathrm{g} / l$, respectively (Table 1). The recoveries were 96-100\%, 99-102\% and $99-112 \%$ for As(III), As(V) and MMA, respectively. Intraday accuracy, expressed as the deviation from the nominal value, was 82.7-99.8\%, and intraday precision, expressed as the relative standard deviation (RSD), was $0.9-7.4 \%$. Interday accuracy and precision were $81.3-100.0 \%$ and $0.8-9.9 \%$, respectively (Table 2). The analytical values of SRM 2669 obtained by the proposed method are shown in Table 3.

\section{Discussion}

Our goal in the present study was to develop and validate a GC-MS method for the simultaneous determination of iAs and MMA in urine. Several GC methods have been reported for the determination of iAs, MMA or DMA ${ }^{8,11-20)}$. In these methods, derivatizations using various thiols, such as $\mathrm{BAL}^{8,11)}$, thioglycol methylate (TGM) ${ }^{12-17)}$ and 1,3-propanedithiol $(\mathrm{PDT})^{18-20)}$, were used to enable GC analyses because these arsenic species are essentially non-volatile. For the choice of an optimal derivatizing reagent, we 

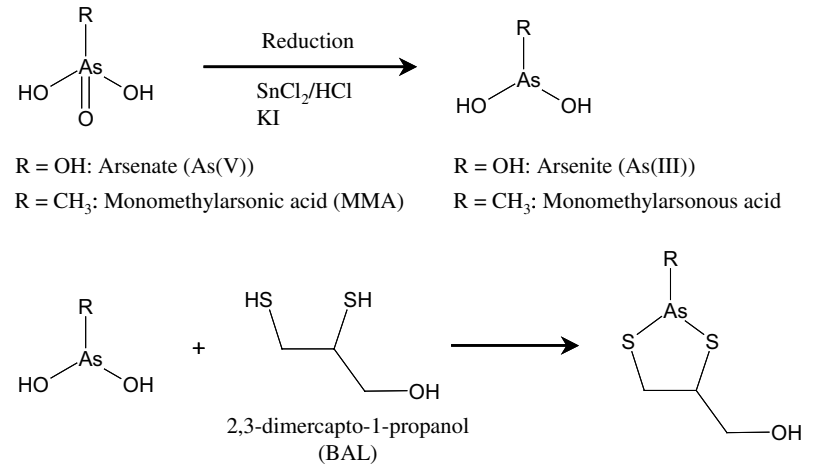

$\mathrm{R}=\mathrm{OH}: \mathrm{BAL}-\mathrm{iAs}$

$\mathrm{R}=\mathrm{CH}_{3}$ : BAL-MMA

Fig. 2. Reaction scheme of inorganic arsenic and monomethylarsonic acid with 2,3-dimercapto-1-propanol as reported by Fukui et al. ${ }^{8)}$

initially examined the use of TGM, but this choice was unsuccessful for iAs. Claussen et al. suggested that an incomplete reaction of iAs with TGM or decomposition of the derivative after injection into the GC system could be the possible reasons for this failure $^{13)}$. Szostek et al. also reported decomposition of the derivative in the GC injector ${ }^{18)}$. We next investigated the use of PDT but did not observe any signal for iAs. In contrast to these derivatizing reagents, BAL showed the best results for iAs and MMA in the derivatization reaction and chromatographic separation. Therefore, we adopted BAL as the derivatizing reagent.

The reaction scheme of iAs and MMA with BAL reported by Fukui et al. is presented in Fig. $2^{8)}$. BAL selectively reacts only with arsenic compounds in the trivalent state to give organic solvent-extractable volatile complexes. Therefore, $\mathrm{As}(\mathrm{V})$ and MMA in their pentavalent state must be reduced to a trivalent state before derivatization. As(III) and As(V) form the same derivative upon reaction with BAL and are determined as the sum of those derivatives. Typical mass chromatograms of blank urine, standard spiked urine and SRM 2669 level II, as well as the mass spectra of BAL-iAs and BAL-MMA, are shown in Fig. 3. The common major fragment ion in both spectra corresponds to $[\mathrm{AsS}]^{+}$at $\mathrm{m} / \mathrm{z} 107$. In the mass spectrum of BAL-MMA, the molecular ion $[\mathrm{M}]^{+}$ of the structure reported by Fukui et al. was observed

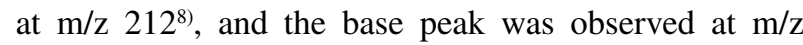
197 , corresponding to the fragment ion of a demethyl derivative $\left(\left[\mathrm{M}-\mathrm{CH}_{3}\right]^{+}\right)$. Other major fragment ions were at $\mathrm{m} / \mathrm{z} 179\left[\mathrm{C}_{3} \mathrm{H}_{4} \mathrm{~S}_{2} \mathrm{As}\right]^{+}$and $165\left[\mathrm{C}_{2} \mathrm{H}_{2} \mathrm{~S}_{2} \mathrm{As}\right]^{+}$. Although the mass spectrum of BAL-MMA agreed with the data of Fukui et al. and the peak of BAL-MMA was assigned on the chromatogram, the peak of BAL-iAs with the mass spectrum reported by Fukui et al. was not observed. However, we found a quantitatively detected peak with a different mass spectrum from that found by Fukui et al. and denoted it as the peak of BAL-iAs. The fragment ions of BAL-iAs reported by Fukui et al. were at $\mathrm{m} / \mathrm{z}$ $214\left([\mathrm{M}]^{+}\right), 212,196,166$ and 107 ${ }^{8)}$, and Siu et al. observed fragment ions of BAL-iAs at m/z 196, 166 and $107^{11)}$. Our mass spectrum showed major fragment ions at $\mathrm{m} / \mathrm{z} 212,165$ and 107 , and the $[\mathrm{M}]^{+}$of the expected structure was not observed, even when GC-MS conditions (temperatures of the injection port and ion source and electron energy) were changed. From the difference of these mass spectra, the peak that we regarded to be BAL-iAs may be different from the presented structure in previous studies. Therefore, further studies (including nuclear magnetic resonance analyses) are in progress to determine the exact structure of BAL-iAs.

The derivatization and extraction procedure was performed according to the method of Fukui et al. with some modifications, including scale-down of the sample volume and change of extraction solvent ${ }^{8)}$. Benzene was used as the extraction solvent in the method of Fukui et al. However, benzene is highly toxic. Therefore, we examined if DCM, toluene, hexane, ethyl acetate or ethyl ether could be used as suitable alternate solvents, as they are less toxic than benzene. DCM and toluene showed almost the same extraction efficiency as that of benzene. Hexane and diethyl ether resulted in poor extraction of BAL-MMA. Ethyl acetate was hardly able to extract BAL-MMA and BAL-iAs. We finally chose DCM as the extraction solvent, because it is less toxic than toluene and gave higher peaks. Moreover, we changed the volume ratio of the sample-extraction solvent from 2 to 4 to obtain higher sensitivity. Details of optimal reaction conditions have not been reported previously. Therefore, we confirmed the influence of the reduction time and the derivatization reaction time. From the obtained results, the optimal reaction conditions were found to be a reduction reaction for 30 minutes and a derivatization reaction for 30 minutes as shown in Fig. 1.

The proposed method was validated according to the US FDA guidelines ${ }^{9}$. The accuracy and precision values of the proposed method met the FDA criteria. The proposed method was further validated by analyzing SRM 2669. The obtained results showed that the proposed method had sufficient accuracy, since the results were not significantly different from the certified values according to application note 1 of the IRMM $^{10)}$ (Table 3). Under the constant GC-MS condition, the robustness of the proposed method was evaluated by using three different columns with the same column size (InertCap 5MS/NP, HP-5MS 

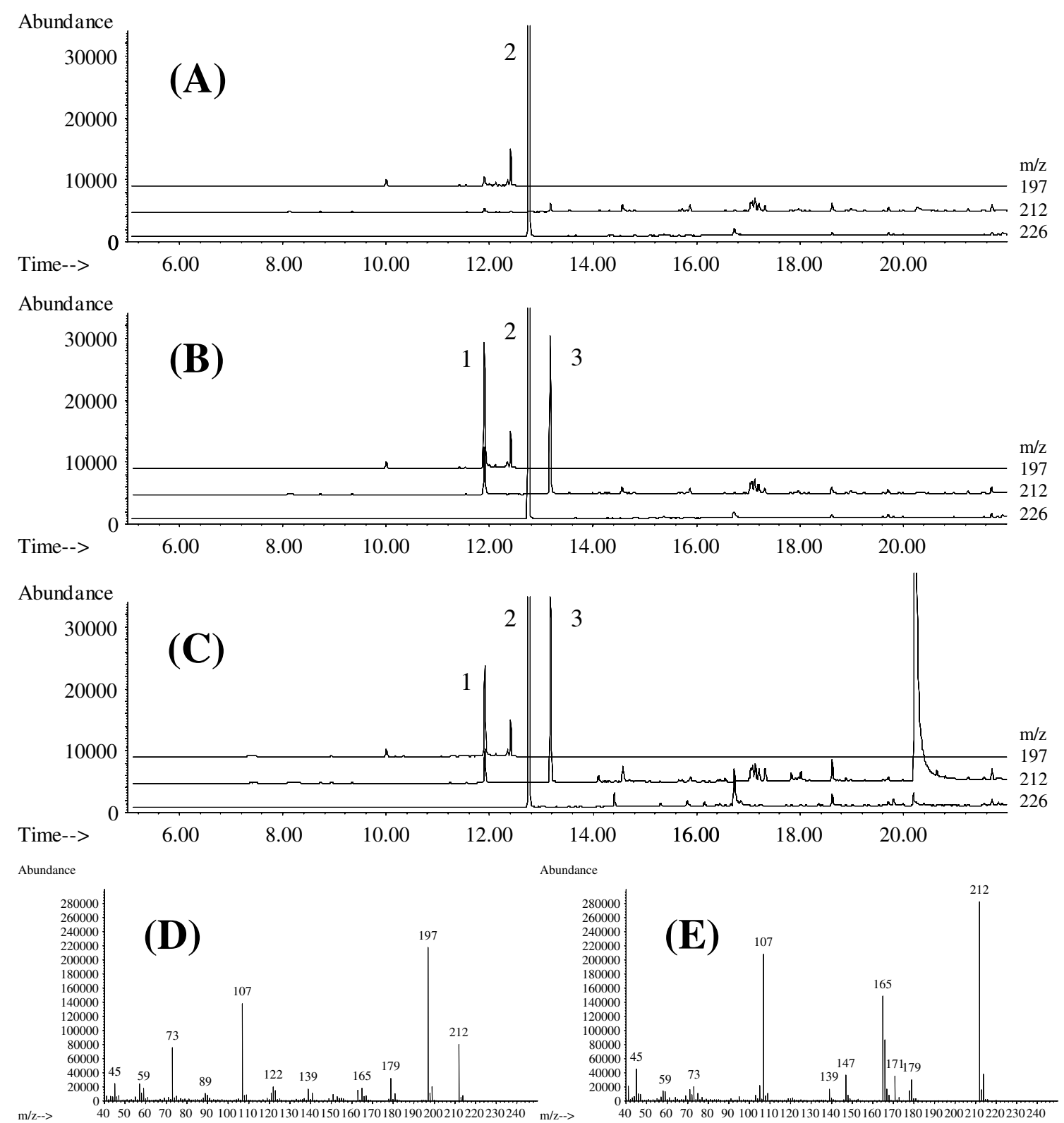

Fig. 3. Typical reconstructed mass chromatograms of a (A) blank urine (MMA, <LOQ; iAs, <LOQ), (B) standard spiked urine (MMA, $10.0 \mu \mathrm{g} / l$; iAs, $10.0 \mu \mathrm{g} / \mathrm{l}$ ) and (C) Standard Reference Material (SRM) 2669 level II (National Institute of Standards and Technology) (MMA, $6.8 \mu \mathrm{g} / l$; iAs, $11.3 \mu \mathrm{g} / l$ ). Typical mass spectra of the derivatives of (D) monomethylarsonic acid (BAL-MMA) and (E) inorganic arsenic (BAL-iAs). Peak 1, BALMMA; peak 2, internal standard (IS); peak 3, BAL-iAs.

and HP-1MS; Agilent Technologies). There were no observed discrepancies in the chromatographic data. Therefore, the proposed method could be considered to be robust.

In comparison with a previously reported HPLCICP-MS method ${ }^{5,7)}$, the proposed GC-MS method not only showed a similar LOD (HPLC-ICP-MS: $0.2-0.3 \mu \mathrm{g} / l)$ but also had several advantages. The proposed GC-MS method had a higher capability of separation for the use of a capillary column and had a higher selectivity and higher capability of identification and confirmation of compounds because mass spectra were obtained. Moreover, it does not require expensive reagents, an expensive and complicated instrument system and high running costs. One of the disadvantages of this method was that it could not distinguish As(III) and As(V). Although a speciation analysis was not required in this study, if necessary, it may be achieved by using a sequential procedure for sample preparation. Another disadvantage was that 
it required a derivatization procedure and a relatively large volume of urine $(2 \mathrm{ml})$. However, requesting a large volume of urine is not a serious problem in routine analyses at periodic health checkups.

In conclusion, we developed and validated a GC-MS method to simultaneously determine iAs and MMA in urine. The proposed method was a robust, selective and cost-effective method suitable for routine analyses and could be useful for the biological monitoring of occupational exposure to iAs.

Acknowledgment: This work was supported in part by a Grant-in-Aid for Scientific Research (B) (No. 23390165) from the Japan Society for the Promotion of Science.

\section{References}

1) International Programme on Chemical Safety (IPCS). Arsenic and arsenic compounds. In: Environmental health criteria 224, 2nd edition. Geneva (Switzerland): World Health Organization (WHO); 2001.

2) American Conference of Governmental Industrial Hygienists (ACGIH). Arsenic and soluble inorganic compounds. In: Documentation of the TLVs and BEIs with other worldswide occupational exposure values, 7th edition. Cincinnati (OH): ACGIH; 2009.

3) Deutsche Forschungsgemeinschaft (DFG). Arsenic and inorganic arsenic compounds. In: Drexler $\mathrm{H}$, Greim H, editors. Essential BAT value documentations. Weinheim (Germany): Wiley-VCH; 2006. p. 77-84.

4) International Agency for Research on Cancer (IARC). Arsenic and arsenic compounds. In: IARC monographs on the evaluation of carcinogenic risks to humans, Volume 100, A review of human carcinogens, Part C: Arsenic, metals, fibres, and dusts. Lyon (France): IARC; 2012. p. 41-93.

5) Hata A, Endo Y, Nakajima Y, et al. HPLC-ICP-MS speciation analysis of arsenic in urine of Japanese subjects without occupational exposure. J Occup Health 2007; 49: 217-23.

6) Ministry of Health, Labour and Welfare (MHLW), Japan. An ordinance to revise the portions of the ordinance on industrial safety and health (Ordinance of the MHLW No. 158 of 2008). Tokyo: MHLW; 2008 (in Japanese).

7) Suzuki Y, Shimoda Y, Endo Y, Hata A, Yamanaka K, Endo G. Rapid and effective speciation analysis of arsenic compounds in human urine using anionexchange columns in HPLC-ICP-MS. J Occup Health 2009; 51: 380-5.

8) Fukui S, Hirayama T, Nohara M, Sakagami Y. Determination of arsenite, arsenate and monomethylarsonic acid in aqueous samples by gas chromatography of their 2,3-dimercaptopropanol (bal) complexes. Talanta 1983; 30: 89-93.

9) U.S. Department of Health and Human Services,
Food and Drug Administration (FDA): Guidance for Industry, Bioanalytical Method Validation. [Online]. 2001 [cited 2012 May 5]; Available from: URL: http://www.fda.gov/downloads/Drugs/Guida nceComplianceRegulatoryInformation/Guidances/ UCM070107.pdf

10) Linsinger $T$, European Commission, Joint Research Centre, Institute for Reference Materials and Measurements (IRMM): Application Note 1, Comparison of a measurement result with the certified value. [Online]. 2010 [cited 2012 May 5]; Available from: URL: http://irmm.jrc.ec.europa. eu/reference_materials_catalogue/user_support/ erm_application_notes/application_note_1/ Documents/erm_application_note_1_english_rev3.pdf

11) Siu KWM, Roberts SY, Berman SS. Derivatization and determination of arsenic in marine samples by gas chromatography with electron capture detection. Chromatographia 1984; 19: 398-400.

12) Dix K, Cappon CJ, Toribara TY. Arsenic speciation by capillary gas-liquid chromatography. J Chromatogr Sci 1987; 25: 164-9.

13) Claussen FA. Arsenic speciation of aqueous environmental samples by derivatization with thioglycolic acid methylester and capillary gas-liquid chromatography-mass spectrometry. J Chromatogr Sci 1997; 35: 568-72.

14) Mester Z, Vitanyi G, Morabito R, Fodor P. Speciation of dimethylarsinic acid and monomethylarsonic acid by gas chromatography-mass spectrometry. J Chromatogr A 1999; 832: 183-90.

15) Mester Z, Horvath G, Vitanyi G, Lelik L, Fodor P. Simultaneous determination of dimethylarsinic acid and monomethylarsonic acid after derivatization with thioglycol methylate by gas chromatography/ mass spectrometry. Rapid Commun Mass Spectrom 1999; 13: 350-3.

16) Mester Z, Pawliszyn J. Speciation of dimethylarsinic acid and monomethylarsonic acid by solid-phase microextraction-gas chromatography-ion trap mass spectrometry. J Chromatogr A 2000; 873: 129-35.

17) Campillo N, Penalver R, Vinas P, Lopez-Garcia I, Hernandez-Cordoba M. Speciation of arsenic using capillary gas chromatography with atomic emission detection. Talanta 2008; 77: 793-9.

18) Szostek B, Aldstadt JH. Determination of organoarsenicals in the environment by solid-phase microextraction-gas chromatography-mass spectrometry. J Chromatogr A 1998; 807: 253-63.

19) Killelea DR, Aldstadt JH, 3rd. Solid-phase microextraction method for gas chromatography with mass spectrometric and pulsed flame photometric detection: studies of organoarsenical speciation. J Chromatogr A 2001; 918: 169-75.

20) Roerdink AR, Aldstadt JH, 3rd. Sensitive method for the determination of roxarsone using solid-phase microextraction with multi-detector gas chromatography. J Chromatogr A 2004; 1057: 177-83. 